\title{
Pheromone Research-Still Something to Write Home About
}

\author{
Joachim Ruther \\ Published online: 12 March 2014 \\ (C) Springer Science+Business Media New York 2014
}

Attending scientific meetings is what gives a researcher's life that extra something. Some years ago, I joined a group of distinguished colleagues for an informal finish of an inspiring conference day at one of the past annual meetings of the International Society of Chemical Ecology (ISCE). We ate together and enjoyed discussions about the situation in our research field. At that time, the study of multitrophic insect-plant interactions was booming after the pioneering findings that plants under herbivore attack combat their plagues by activating direct defenses and attracting natural enemies. Not an easy time for pheromone researchers as illustrated by the statement made by one of the names at our table: "Today, pheromone research is nothing to write home about anymore." This was a harsh statement arousing my opposition, but I wasn't sure whether my defensive arguments convinced my opinionated counterpart; I was still at an early stage of my scientific career. In 2014, the Journal of Chemical Ecology, beyond doubt one of the most important forums for the publication of pheromone-related research, celebrates its 40th anniversary, presenting me a good opportunity to provide some topical counter-arguments.

In the early years of chemoecological research, identification of the first lepidopteran sex pheromones raised hopes that pheromones and other semiochemicals might revolutionize pest control, and early chemoecologists hoped that pheromones might even replace pesticides in the long run. Today, it is clear that this optimism was somewhat premature, because catching or disorientating insects does not necessarily result in the regulation of their populations, nor the protection of plants and agricultural products, respectively. However, a recent review by Witzgall et al. (2010) published in this journal compiled numerous examples demonstrating that semiochemical-based pest control is working in principle. Apart from this, pheromones offer hitherto underestimated possibilities that can be applied in nature conservation. Rare species, for instance, can be monitored by pheromone traps, and cryptic species can be detected by analyzing their pheromone composition. Therefore, studies that identify novel natural products and test their applicability in environmentally sound pest control and nature conservation will remain important future tasks of chemoecologists. The contribution of applied pheromone research to the sustainable use of ecosystem services is not the least strong argument when communicating chemoecological research to funding agencies and the general public.

Modern pheromone research, however, is much more than asking what pheromones are composed of and how we can exploit them to control insect pests. Pheromone research has drastically broadened its scope, and apart from the obvious proximate questions it has been shown that pheromones possess an enormous potential for studying evolutionary questions concerning intraspecific communication (Smadja and Butlin 2009). Pheromones are intraspecific chemical signals and as such both the sender and the receiver must have evolved to use the chemical messengers for the exchange of information with conspecifics. However, what qualifies a given compound to be used as a chemical signal, and how do pheromone biosynthesis and perception co-evolve? Does pheromone divergence initiate or finalize speciation, or does it merely minimize the costs of

\section{J. Ruther $(\square)$}

Institute of Zoology, University of Regensburg, Universitätsstraße 31, 93053 Regensburg, Germany e-mail: joachim.ruther@biologie.uni-regensburg.de interspecific mating after speciation has been completed? We are only beginning to understand the selective forces and the genetic mechanisms underlying pheromone diversification in a few model organisms. The possible link between physiological and ecological costs of chemical signals and the maintenance of signal honesty needs further investigation. In the context of sexual communication, it becomes increasingly clear that pheromones do not merely transfer information concerning the presence of a potential mate but also enable the receiver to assess mate quality and to choose a mate accordingly (Johansson and Jones 2007). All these questions, of course, are not completely new to chemoecologists, but I believe that the potential of pheromones in this respect has not yet been tapped and deserves our increased future attention.

Hitherto, many aspects concerning the involvement of intraspecific signals in evolutionary processes have been studied preferentially in species communicating via visual or auditory signals. However, pheromones have many advantages compared to signals perceived by other sensory modalities. The versatile toolbox of analytical chemistry enables us to describe precisely both the quality and the quantity of a chemical signal, and thus to specify the variability of chemical information within and between different populations of a given species. Today, even the pheromone doses released by individual animals can be monitored in real time. Hence, the strength of a chemical signal and its composition can be linked directly to an individual's quality parameters and mating success. By using stable isotope labeling techniques, we can identify pheromone precursors, which may enable us to establish a causal link between nutritional parameters and chemical signaling as well as to evaluate the physiological costs of pheromone production. The achievements of synthetic organic chemistry allow us to mimic exactly or manipulate chemical signals and thus to directly demonstrate the involvement of a given chemical messenger in biological processes rather than relying merely on correlations.

Last but not least, the highly sophisticated tools of biochemistry, molecular biology, and neurophysiology are being increasingly used to study the mechanisms underlying pheromone biosynthesis and perception, as well as to characterize sequences and expression patterns of the genes involved. The increasing number of model organisms with sequenced genomes and known pheromone composition should accelerate the advances in this respect.

In conclusion, pheromone research is and will remain "something to write home about," but due to the increasing complexity of questions and techniques it will presumably become more difficult for those staying at home to understand the reports. Pheromones are predestined for the study of evolutionary aspects in intraspecific communication because they offer excellent opportunities to link ultimate questions with the underlying proximate mechanisms. Both perspectives are indispensable for a comprehensive understanding of biological processes.

\section{References}

Johansson BG, Jones TM (2007) The role of chemical communication in mate choice. Biol Rev 82:265-289. doi:10.1111/j.1469-185X.2007.00009.x

Smadja C, Butlin RK (2009) On the scent of speciation: the chemosensory system and its role in premating isolation. Heredity 102:77-97. doi:10. 1038/hdy.2008.55

Witzgall P, Kirsch P, Cork A (2010) Sex Pheromones and their impact on pest management. J Chem Ecol 36:80-100. doi:10.1007/s10886-009-9737-y 